\title{
ON THE LONG-WAVELENGTH TRANSITION RADIATION BY RELATIVISTIC ELECTRONS OBLIQUE MOVING THROUGH A THIN TARGET
}

\author{
S.N. Dobrovolsky ${ }^{\dagger}$, N.F. Shul'ga* \\ N S C "Kharkov Institute of Physics and Technology", Kharkov, 61108, Ukraine
}

\section{Abstract}

The transition radiation produced by relativistic electron on the thin metallic transverse-bounded target for the case of oblique electron transit is investigated. We show that as well as for the case of normal electron transit the intensity of "backward" and far-field "forward" radiation are strongly distorted in some region of waves. The condition of distortion is defined by ratio between transversal size of target and effective transversal diameter of radiation formation region.

\section{INTRODUCTION}

The long-wavelength transition radiation from bunched relativistic electrons is increasingly utilised as a goon method for non-destructive bunch testing and as a source of quasi-monochromatic infrared radiation. Formation of ultra-short bunches of electrons let to observe the coherent transition radiation in the millimeter range of waves. It is well known fact that in the relativistic regime the longitudinal length (along a direction of electron velocity) of region where radiation is formed can reach some tens of meters. In this condition the effect of interference between "forward" radiation and own electron field in the "formation region" is possible. But under these conditions the transversal diameter of "formation region" may be a macroscopic value also (up to ten centimetres) and compared with transversal size of used targets. So, the influence of transversal bounds of target to the transition radiation intensity is expected in this case. This effect is kept in force for radiation by a single relativistic electron. Recently we proposed the theory of transition radiation of relativistic electron on the thin transverse bounded target in the case of normal electron transit [1-2]. Now we consider the general case of oblique transition of electron through a target.

The special interest is connected with case of producing transition radiation by relativistic electron in the inclined thin metallic screen. The "forward" "backward" radiation is equal in this case. The "backward" radiation is not directed to the electron's trajectory and it is well-detected at the arbitrary distances.

\footnotetext{
*shulga@kipt.kharkov.ua

† dobrovolsky@kipt.kharkov.ua
}

\section{THEORY}

\subsection{Radiation fields}

Consider the transition radiation emitted by relativistic electron oblique passing through a center of thin metallic disc. We assume that disk is fairly thin, i. e. the thickness of target is greatly less than radiated wavelength but greater than "penetration length" of electron field into metallic target. Let's the electron moved in a XOZ plane. It can be assumed that the field not incident on the target is diffracted at an infinitely thin screen of a radius $a$ positioned in the plane $z=0$. After the electron has left the target the electric fields $\mathbf{E}^{+}(\mathbf{r}, t)$ and $\mathbf{E}^{-}(\mathbf{r}, t)$ in space $z>0$ and $z<0$ accordingly may be written in the form

$$
\begin{gathered}
\mathbf{E}^{+}(\mathbf{r}, t)=\mathbf{E}^{(e)}(\mathbf{r}, t)+\mathbf{E}^{(+)}(\mathbf{r}, t), \\
\mathbf{E}^{-}(\mathbf{r}, t)=\mathbf{E}^{\prime(-)}(\mathbf{r}, t),
\end{gathered}
$$

where $\mathbf{E}^{(e)}(\mathbf{r}, t)$ is a own electron field and $\mathbf{E}^{\prime \pm)}(\mathbf{r}, t)$ is a "forward" and "backward" radiation fields. The Fourier component of the $\mathbf{E}^{(e)}(\mathbf{r}, t)$ field with respect to time of an electron propagating uniformly at velocity $\mathbf{v}$ in vacuum on the angle $\phi$ to the axis $\mathrm{OZ}$ is determined by form (we neglected the terms of order $\gamma^{-2}$ )

$$
\begin{aligned}
\mathbf{E}_{\omega}^{(e)}(z, \boldsymbol{\rho}) & \approx \frac{e \omega}{v_{z}^{2} \gamma} 2 K_{1}\left(\frac{\omega \rho}{v \gamma} \sqrt{1+\sin ^{2} \phi \frac{z^{2}-x^{2}}{\rho^{2}}-\sin 2 \phi \frac{z x}{\rho^{2}}}\right) \times \\
& \frac{\boldsymbol{\rho}-\sin \phi\left(\mathbf{e}_{x}[z \cos \phi+x \sin \phi]+\mathbf{e}_{z}[x \cos \phi-z \sin \phi]\right)}{\rho \sqrt{1+\sin ^{2} \phi \frac{z^{2}-x^{2}}{\rho^{2}}-\sin 2 \phi \frac{z x}{\rho^{2}}}} \times \\
& \exp \left(i \frac{\omega}{v_{z}}(z \cos \phi+x \sin \phi)\right),
\end{aligned}
$$

where $v_{z}$ is a OZ-projection of electron velocity, $e$ is a electron charge, $\gamma$ is a Lorentz-factor of electron. Also, $K_{1}$ is a McDonald first-order function and $\boldsymbol{\rho}=x \mathbf{e}_{\mathbf{x}}+y \mathbf{e}_{\mathbf{y}}, \mathbf{e}_{\mathbf{x}}$ и $\mathbf{e}_{\mathbf{y}}$ - unitary vector along $O X$ and $O Y$ axis.

The "forward" and "backward" radiation fields $\mathbf{E}^{\prime( \pm)}(\mathbf{r}, t)$ are propagate in positive and negative direction of $\mathrm{OZ}$ axis as a wave packet of free electromagnetic waves. Because the target is a ideal metallic screen in our conditions the radiation fields are 
satisfy to the next conditions on the plane of screen $z=0$

$$
\begin{gathered}
\mathbf{E}_{\perp}^{\prime( \pm)}(\mathbf{r}, t)=\Theta(a-\rho) \mathbf{E}_{\perp}{ }^{(e)}(\mathbf{r}, t) \\
\mathbf{E}_{\mathrm{II}}^{\prime( \pm)}(\mathbf{r}, t)=-\Theta(a-\rho) \mathbf{E}_{\mathrm{II}}{ }^{(e)}(\mathbf{r}, t),
\end{gathered}
$$

where $\mathbf{E}_{\mathrm{II}}^{\prime \pm)}(\mathbf{r}, t)$ и $\mathbf{E}_{\perp}^{\prime( \pm)}(\mathbf{r}, t)$ are the parallel and orthogonal to the target's plane part of radiation fields, $\Theta(x)$ - Heaviside step function $(\Theta(x)=1$, if $x \geq 0$ and $\Theta(x)=0$, if $x<0), \mathbf{r}=\boldsymbol{\rho}+z \cdot \mathbf{e}_{\mathbf{z}}, \mathbf{e}_{\mathbf{z}}-$ unitary vector of $O Z$ axis.

Now, by taking into account the (2) and (3) equations we obtain the following expression for the transversal spatial Fourier components of radiation fields

$$
\begin{aligned}
& E_{\omega, \chi}^{\prime( \pm)}=\frac{2 e \omega}{v_{z}^{2} \gamma} \int_{-\pi}^{\pi} d \alpha \int_{0}^{a} \rho d \rho\left[ \pm \cos \alpha \cos \phi\left(\vec{e}_{x} \cos \phi+\vec{e}_{z} \sin \phi\right)+\vec{e}_{y} \sin \alpha\right] \times \\
& \times K(\rho, \phi, a) \exp \left(-i\left[\chi \rho \pm z \sqrt{k^{2}-\chi^{2}}-\frac{\omega \rho}{v_{z}} \cos \alpha \sin \phi\right]\right) \\
& \text { where } K(\rho, \phi, \alpha)=\frac{K_{1}\left(\frac{\omega \rho}{v_{z} \gamma} \sqrt{1-\sin ^{2} \phi \cos ^{2} \alpha}\right)}{\sqrt{1-\sin ^{2} \phi \cos ^{2} \alpha}}
\end{aligned}
$$

and $\chi=\omega c^{-1} \sin \vartheta$.

\subsection{Spectral-angular density of radiation}

We shall now consider an energy flux passing across some infinite plane $z=z_{0}$ (detector) over the entire observation time. For "forward" radiation we assume that $z_{0}$ is greater than "formation length", i.e., we located in "wave zone". The projection of the Poynting vector on the $\mathrm{OZ}$ axis is given

$$
S_{z}=\frac{c}{(2 \pi)^{4}} \int_{\omega>0} d \omega \int d \boldsymbol{\chi}\left|\mathbf{E}_{\omega, \chi}^{\prime( \pm)}\left(z_{0}\right)\right|^{2} \cos \vartheta
$$

Expanding the fields (4) into Fourier integrals for spectral-angular density on a unit of frequency and on the unit of solid angle $d \Omega=\sin \vartheta d \vartheta d \varphi$ of "forward" and "backward" transition radiation we obtain the next expression (the $\vartheta$ angle is counted out negative and positive directions of OZ-axis for "backward" and "forward" radiation correspondingly)

$$
\begin{gathered}
\frac{d^{3} S_{z}^{( \pm)}}{d \omega d \Omega}=\frac{d^{3} S_{z}^{(\infty)}}{d \omega d \Omega} \cdot A^{2}+ \\
+\frac{e^{2}}{\pi^{2} c} \frac{\beta^{2} \cos ^{2} \phi \sin ^{2} \vartheta \cos ^{2} \vartheta \sin ^{2} \varphi \cdot\left(B^{2}-A^{2}\right)}{\left([1-\beta \sin \phi \sin \vartheta \cos \varphi]^{2}-\beta^{2} \cos ^{2} \vartheta \cos ^{2} \phi\right)^{2}},
\end{gathered}
$$

where

$$
\begin{gathered}
\frac{d^{3} S_{z}^{(\infty)}}{d \omega d \Omega}=\frac{e^{2}}{\pi^{2} c} \beta^{2} \cos ^{2} \phi \frac{(\sin \vartheta-\beta \sin \phi \cos \varphi)^{2}+\beta^{2} \sin ^{2} \phi \cos ^{2} \vartheta \sin ^{2} \varphi}{\left((1-\beta \sin \phi \sin \vartheta \cos \varphi)^{2}-\beta^{2} \cos ^{2} \vartheta \cos ^{2} \phi\right)^{2}} \\
A=\frac{I(x, \vartheta, \varphi, \phi)}{I(\infty, \vartheta, \varphi, \phi)}, \quad B=\frac{J(x, \vartheta, \varphi, \phi)}{J(\infty, \vartheta, \varphi, \phi)}
\end{gathered}
$$

and

$$
\begin{gathered}
I(u, \vartheta, \varphi, \phi)=\int_{-\pi}^{\pi} d \alpha \int_{0}^{u} t d t \cdot K(t, \phi, \alpha) \cdot \cos \alpha \cdot Q(t, \alpha, \vartheta, \varphi, \phi) \\
J(u, \vartheta, \varphi, \phi)=\int_{-\pi}^{\pi} d \alpha \int_{0}^{u} t d t \cdot K(t, \phi, \alpha) \cdot \sin \alpha \cdot Q(t, \alpha, \vartheta, \varphi, \phi) \\
Q=\exp [-i t \gamma \cos \alpha(\sin \vartheta \cos \varphi-\beta \sin \phi)-i t \gamma \beta \sin \vartheta \sin \varphi \sin \alpha] \\
K(t, \phi, \alpha)=\frac{K_{1}\left(t \sqrt{1-\sin ^{2} \phi \cos ^{2} \alpha}\right)}{\sqrt{1-\sin ^{2} \phi \cos ^{2} \alpha}}, \quad x=\frac{\omega a}{v_{z} \gamma}
\end{gathered}
$$

The formula (6) is describe the spectral-angular density of energy flux across a some z-plane (detector) for "forward" and "backward" transition radiation occurred after oblique transition of relativistic electron through a thin metallic disc.

\subsection{Discussion}

The spectral-angular density of transition radiation (6) is composed out of spectral-angular density of radiation on infinite metallic plate $\frac{d S_{z}^{(\infty)}}{d \omega d \Omega}$ and some functions $A$ and $B$ which are responsible for influence of bounded transversal size of target. These functions are depends from parameter $x=\frac{\omega a}{v_{z} \gamma}$. This parameter is a ratio between effective transversal diameter of formation region and transversal size of target.

Now, let's consider the general case of non-small angle $\phi$ (the angle $\phi$ is an angle between electron's velocity and normal vector to the target's plane). We assume also that $\phi$ is not nearly $\pi / 2$, i.e. we not consider the case of sliding incidence of electron

At the frequency range $\omega \gg v_{z} \gamma a^{-1}$ we have $A \rightarrow 1, B \rightarrow 1$ and the formula (6) is a well-known expression for a radiation on the infinite target occurred after electron oblique passing [3]. This case situated when the effective transversal sizes of own electron field (diameter of formation region) is sufficiently less than target's diameter.

But in the range where $\omega \leq v_{z} \gamma a^{-1}$ the functions $A$ and $B$ not be equal to unity and it's have strong oscillations and rapidly decreased to the zero at $\omega<<v_{z} \gamma a^{-1}$ (see for example fig.1 - the graph for $A$ at simplest case $\varphi=0)$. 


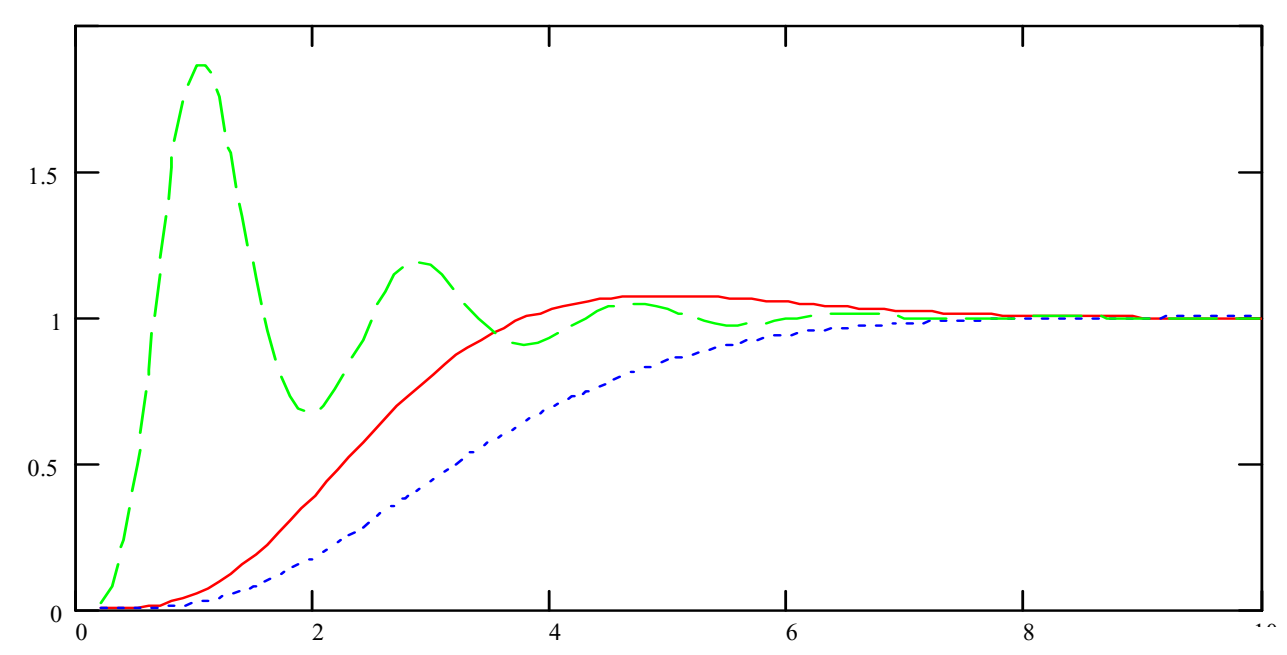

Figure 1: Function $\Phi=A^{2}(x, \vartheta, \varphi, \gamma, \phi)$ from $x=\frac{\omega a}{v_{z} \gamma}$ for the various values of $\vartheta$ under $\phi=\pi / 4, \gamma=200, \varphi=0$. Here solid line corresponds to $\vartheta=\phi+\gamma^{-1}$, dashed line $-\vartheta=\phi+5 \gamma^{-1}$ and dotted line $-\vartheta=\phi+0.5 \gamma^{-1}$.

Correspondingly, the spectral-angular intensity of radiation in this case will distort at comparison with intensity of transition radiation on infinite screen. The main angular maximum is moved to the greater angles and value of intensity is decreased.

In the case of $\phi \rightarrow 0$ we have $A \approx B \rightarrow F$, where $F=F(\gamma \vartheta, x)$ - is the function of influence transverse size of target for the normal electron incident case. For this we obtain the next spectral-angular density of transition radiation [1-2]

$$
\begin{gathered}
\frac{d S}{d \omega d \Omega}=\frac{e^{2}}{\pi^{2} c} \frac{\sin ^{2} \vartheta \cos ^{2} \vartheta}{\left(\sin ^{2} \vartheta+\gamma^{-2}\right)^{2}} F^{2}(\gamma \sin \vartheta, x) \\
F(y, x)=\frac{y^{2}+1}{y} \int_{0}^{x} u d u K_{1}(u) J_{1}(y u) .
\end{gathered}
$$

\section{CONCLUSIONS}

We have considered the problem of relativistic electron's transition radiation that occurred after oblique transition of electron through a thin metallic disk. The formulas of spectral-angular density of radiation that describes "forward" and "backward" radiation intensity and take into account the bounded transversal size of target are obtained.
At arbitrary angles of electron incidence we have identically formulas as for "forward" radiation in the wave zone as well as for "backward" radiation. In contrast to case of normal incidence these expressions are more complex and depend from ratio between transversal target size and diameter of "formation region" of radiation.

The transversal bounding of target is considered by two functions. We observed the strong effect of target's transversal size influence to the spectral-angular density of radiation in the range of radiated waves where $\omega \leq a^{-1} v_{z} \gamma$. In compare with case of infinite target plate, the angular distribution of transition radiation is distorted and its intensity can decrease by one or more degree. For relativistic electrons and ten-centimetres targets its effect is occurred in the millimetres and submillimetres range of waves.

\section{REFERENCES}

[1] N.F. Shul'ga, S.N. Dobrovolsky JETP, V.90, No.4, 2000, pp. 579-583

[2] N.F. Shul'ga, S.N. Dobrovolsky Phys. Lett A 259, 1999, pp. 291-294.

[3] M.L. Ter-Mikaelyan "High-Energy Electromagnetic processes in Condensed Media", Wiley Int. NewYork, 1972. 